\title{
Article
}

\section{The disengage deficit in hemispatial neglect is restricted to between-object shifts and is abolished by prism adaptation}

Schindler, I., Mclntosh, R.D., Cassidy, T.P., Birchall, D., Benson, Valerie, letswaart, M. and Milner, A.D.

Available at http://clok.uclan.ac.uk/28072/

Schindler, I., McIntosh, R.D., Cassidy, T.P., Birchall, D., Benson, Valerie ORCID: 0000-0002-0351-4563, letswaart, M. and Milner, A.D. (2009) The disengage deficit in hemispatial neglect is restricted to between-object shifts and is abolished by prism adaptation. Experimental brain research, 192 (499). ISSN 0014-4819

It is advisable to refer to the publisher's version if you intend to cite from the work. http://dx.doi.org/10.1007/s00221-008-1585-4

For more information about UCLan's research in this area go to http://www.uclan.ac.uk/researchgroups/ and search for <name of research Group>.

For information about Research generally at UCLan please go to http://www.uclan.ac.uk/research/

All outputs in CLoK are protected by Intellectual Property Rights law, including Copyright law. Copyright, IPR and Moral Rights for the works on this site are retained by the individual authors and/or other copyright owners. Terms and conditions for use of this material are defined in the policies page. 

Submitted to Experimental Brain Research for the special issue in honour of Professor G Berlucchi

The disengage deficit in hemispatial neglect is restricted to between-object shifts and is abolished by prism adaptation

I. Schindler ${ }^{1,2}$, R.D. McIntosh ${ }^{1,3}$, T.P. Cassidy ${ }^{4}$, D. Birchall ${ }^{5}$ V Benson ${ }^{1,6}, \mathrm{M}$ Ietswaart $^{1,7}$, and A.D. Milner ${ }^{1}$

${ }^{1}$ Cognitive Neuroscience Research Unit, Wolfson Research Institute, Durham University, Queen's Campus, Stockton-on-Tees TS17 6BH, UK.

${ }^{2}$ Department of Psychology, University of Hull, Hull HU6 7RX, UK.

${ }^{3}$ Human Cognitive Neuroscience, University of Edinburgh, Edinburgh EH8 9JZ, UK. ${ }^{4}$ Stroke Medicine Department, Queen Elizabeth Hospital, Gateshead, Tyne and Wear NE9 6SX, UK

${ }^{5}$ Division of Neuroradiology, Regional Neurosciences Centre, Newcastle General Hospital, Westgate Road, Newcastle-upon-Tyne NE4 6BE

${ }^{6}$ School of Psychology, University of Southampton, Southampton SO17 1BJ

${ }^{7}$ School of Psychology and Sport Sciences, Northumbria University, Newcastle-uponTyne NE1 8ST.

Corresponding author:

Professor A D Milner

Wolfson Research Institute

Durham University Queen's Campus

Stockton-on-Tees TS17 6BH, UK.

a.d.milner@durham.ac.uk; phone +44-191-334-9148; fax +44-191-334-3241.

Keywords: neglect, prism adaptation, attention, within-object, Posner. 


\begin{abstract}
We sought to determine the effects of prism adaptation on peripherally-cued visual attention shifting in patients with spatial neglect, using a task devised by Egly and colleagues (1994) based on the classic Posner paradigm. This task allowed a comparison of "within-object" versus "between-object" attention shifts. A display was presented containing two parallel outline rectangles, and subjects were asked to make rapid responses to a target, which would appear at one end of one of the rectangles. The target location was pre-cued with $75 \%$ validity: on invalid trials attention was directed either to the other end of the same rectangle, or to the other rectangle. Healthy subjects and right-hemisphere patients without neglect showed a left-right symmetrical pattern, with a larger validity effect when required to shift attention between rectangles, thus indicating a greater difficulty of attention-shifting between than within the respective shapes. The neglect patients showed the typical leftward 'disengage deficit' previously observed in neglect, but only for attention shifts between objects, indicating that the effect is object-based rather than purely spatial. A comparison of vertical and horizontal shift costs showed that this attentionshifting deficit for left-hemifield target stimuli was directional rather than hemifieldbased: it was absent for vertical shifts of attention within the left hemifield. Finally, we found that prism adaptation abolished the disengage deficit. We found no effects of prism adaptation in the control subjects. We argue that prism adaptation has a powerful effect on one of the fundamental manifestations of the neglect syndrome.
\end{abstract}




\section{Introduction}

A powerful and simple technique for temporarily modifying the symptoms of unilateral spatial neglect was reported by Rossetti and colleagues (1998). They found that a session of a few minutes in which the patient repeatedly made pointing responses to a simple target while wearing $10^{\circ}$ rightward-displacing prismatic goggles was sufficient to cause quite major improvements across a wide range of symptoms. The tasks showing improvement included standard clinical tests such as cancellation, copying and bisection. Moreover subsequent studies have demonstrated that the effects may generalize to wheelchair navigation (Rossetti et al. 1999), postural control (Tilikete et al. 2001), neglect of mental imagery (Rode et al. 1999, 2001) and haptic spatial judgements (McIntosh et al. 2002). The observations indicate that visuomotor adaptation causes a temporary reorganization of the neural representation of space that persists for hours or days following removal of the prisms (Milner and McIntosh 2005). The way in which this change occurs, however, remains obscure (see Redding and Wallace 2006, for a discussion of possible mechanisms).

One plausible hypothesis would be that visuomotor prism adaptation causes a short-term redistribution of attentional resources across visual space. The evidence for such a proposal, however, is mixed. In favour of it is the finding by Berberovic et al. (2004) that the ipsilesional attentional bias of neglect patients on a left/right visual temporal order judgement task was significantly reduced following adaptation. In a second study by the same research group, however, Morris and colleagues (2004) reported no effect of adaptation on the steep lateral gradients of visual search performance that are typical of, and arguably a more central feature of, neglect. 
A more direct test of the attention-redistribution hypothesis would be to use the well-known attentional cueing procedure devised by Posner (1980). Several previous studies have shown that neglect patients experience an exaggerated cost for shifts of attention toward the contralesional side when given an invalid ipsilesional cue (Morrow and Ratcliff 1988; Ladavas et al. 1994; Losier and Klein 2001). The initial studies selected patients on an anatomical rather than a diagnostic basis (e.g. Posner et al. 1982, 1984, 1987; Friedrich et al. 1998) and found this so-called 'disengage deficit' in both left and right parietal-lesioned patients. In a meta-analysis, however, Losier and Klein (2001) found a much bigger deficit in parietal patients with neglect than in those without.

Two recent small-scale studies have used the Posner paradigm specifically to examine the effects of prism adaptation in neglect patients. First, Striemer and Danckert (2007) tested four right-brain damaged patients, two of them with neglect, on an exogenous version of the Posner task (i.e. using non-informative, peripheral cues). They reported that prism adaptation reduced both the rightward attentional bias and the disengage deficit, irrespective of the presence of neglect, especially at a short SOA between cue and target $(50 \mathrm{~ms})$. In the other study, however, Nijboer et al. (2008) reported data on two neglect patients suggesting a contrary conclusion, namely that voluntary (i.e. endogenous) shifts of attention benefit from prism adaptation while exogenous shifts do not.

In the present paper we report a much more extensive study of the effects of prism adaptation on attentional orienting, testing groups of both neglect patients and right-brain damaged controls, as well as a group of healthy controls. We were interested in determining whether there is an object-based component to the disengage deficit in neglect, or whether it depends simply on spatial factors. To this end, we 
adopted a task from Egly and colleagues (1994), designed to contrast the shifting of attention between two objects as opposed to two locations within a single objects. These investigators presented subjects with a visual array containing two parallel outline rectangles, and asked them to make rapid responses to a target, which would appear at one end of one of the rectangles. The target location was pre-cued with $75 \%$ validity, and invalid trials either directed attention to the 'wrong' end of the correct rectangle, or to the 'right' end of the incorrect rectangle. Normal subjects showed a larger validity effect for attention shifts between rectangles, indicating an objectbased component of attention. The authors also tested patients with left or right parietal lesions, and reported, as expected, that both groups showed elevated costs of invalid cueing for contralesional compared with ipsilesional targets in the betweenobject paradigm. This high cost for attention shifts toward objects on the contralesional side was significantly greater in the left-hemisphere patients than for shifts made within objects. However, the right-hemisphere patients showed no such differential cost for between versus within-object shifts to the contralesional side, suggesting that their disengage deficit was purely spatial. In agreement with Egly et al.'s (1994) data, there is accumulating evidence that object-based neglect is more common in patients whose neglect results from left- rather than right-hemisphere damage (Kleinman et al. 2007).

We adopted an essentially identical experimental procedure in the present study, but used it specifically to compare groups of right-hemisphere patients either with or without left visuospatial neglect. Our aim was to discover whether betweenobject shifts toward left-side stimuli would be subject to greater reaction-time costs than within-object shifts in our patients with neglect, and thus whether there is an object-based component to the disengage deficit in neglect. Our second aim was to 
assess the extent to which performance in these two versions of the Posner paradigm would be amenable to modification by prism adaptation.

\section{Methods}

\section{Subjects}

In total, 32 right-handed subjects participated in the experiment (see Table 1 for clinical and demographic details). The neglect group (NEG) consisted of 10 right brain-damaged patients with a mean age of 68.3 years ( $\mathrm{SD}=10.9$ years), all of them diagnosed clinically as having spatial neglect. Ten right brain-damaged patients without neglect served as neurological controls (RBD: mean age $=66.2$ years, $\mathrm{SD}=$ 10.8 years). None of the patients suffered from visual field defects as assessed by finger perimetry, general mental deterioration as clinically assessed, or psychiatric disorder. In addition, the study included 12 healthy, age matched controls (HC) without any history of neurological damage (mean age $=68.7$ years, $\mathrm{SD}=7.3$ years). All subjects were right-handed and gave their informed consent prior to their inclusion in the study. The study was conducted in accordance with the ethical standards laid down in the 1964 Declaration of Helsinki, and was approved by the NHS regional ethics committee (MREC).

\section{Neglect tests}

The presence of neglect was determined by impaired performance on at least two of the following five standard neglect screening tests: two visual search tasks - star cancellation from the Behavioural Inattention Test (BIT: Wilson et al. 1987), and the Balloons Test version-B (Edgworth et al. 1998); line bisection (BIT); scene copying 
(Gainotti et al. 1972); and drawing from memory. For star cancellation, the number of targets omitted on each side was counted, and neglect was defined by a cut-off score of $\leq 51$ and at least $10 \%$ more omissions of targets on the left than on the right. For the Balloons test an index of left sided inattention was calculated based on the proportion of targets found on the left side relative to the total number of found targets [(Left/Total)*100]. The cut off value was an index of $<45 \%$. Performance in the line bisection task was measured by calculating the average deviation of the bisection marks from the objective centre of each line in mm, with leftward deviations scored as negative and rightward deviations scored as positive. The cut off score for a significant rightward bias was $>+12.5 \mathrm{~mm}$, corresponding to $>5 \%$ of the total line length. Scene copying was scored according to the number of objects copied (total $n=$ 5) and the number of objects symmetrically depicted, with neglect diagnosed by the omission or transposition of any objects at the left of the picture and/or omission of left-sided details from any of the attempted items. In the drawing task each patient was asked to draw a daisy, a clock face, and a person. The quality of the drawings was scored based on symmetry with a maximum score of 3 for all three items depicted symmetrically and a minimum score of 0 for no item depicted symmetrically. Neglect was inferred if left-sided details were omitted or transposed from one or more of the drawings.

Table 1 about here

\section{Prism adaptation}

For the prism adaptation procedure subjects wore a pair of goggles fitted with widefield prismatic lenses inducing a rightward optical shift of $10^{\circ}$. Exposure consisted of 
200 fast pointing movements made to visual targets (dots) presented either $10^{\circ}$ to the left or right of the body midline, at a distance of $65 \mathrm{~cm}$, with 100 responses made to each in pseudo-random order. A board was positioned horizontally under the subject's chin to conceal the hand from view at its starting position but allowing an unobstructed view of the targets and terminal pointing errors. The sham adaptation procedure was identical except that plain, uncorrected lenses were used. After removal of the goggles (whether prism or sham), subjects made four fast open-loop pointing movements to a central target dot on the table to verify the presence of a prismatic after-effect. Open-loop pointing was re-tested at the end of each trial block to confirm that the after-effects were still present. These pointing errors were formally recorded (manually, in $\mathrm{cm}$ from the target) only for the prism condition, immediately after the adaptation trials (before the attention task) and at the end of the session (after the last block of trials).

\section{Attentional Paradigm}

Subjects were seated in a darkened room in front of a 17-inch computer monitor (resolution 1027 x 768 pixels). The viewing distance was $57 \mathrm{~cm}$, with the centre of the screen at eye level. The subject's head position was controlled by a chin-rest. At the beginning of each trial, four white, solid squares $\left(0.4^{\circ} \times 0.4^{\circ}\right)$ would converge, over $1000 \mathrm{~ms}$, from the corners of a virtual square $(40 \mathrm{~mm})$ at the centre of a black screen (see Fig. 1) to draw gaze to this location. At the point of convergence, the square was replaced by a white central fixation cross $\left(0.4^{\circ} \times 0.4^{\circ}\right)$ along with two grey rectangles, either horizontally or vertically aligned. Each rectangle subtended $2.2^{\circ} \mathrm{x}$ $15.2^{\circ}$ with a line thickness of $0.1^{\circ}$, and was centred $4.5^{\circ}$ from fixation. 
After $1000 \mathrm{~ms}$ a peripheral pre-cue was presented in the form of a brightening (grey to white) of three sides of an imaginary outline square $\left(2.2^{\circ}\right)$, which overlapped one end of the rectangle. After $100 \mathrm{~ms}$ the cued end returned to its original grey colour and the fixation display remained on-screen for another $100 \mathrm{~ms}$. This was followed by the target, a solid grey square $\left(0.2 \times 0.2^{\circ}\right)$. The target could appear at either the cued end of the rectangle (valid trial), the opposite end of the same rectangle (invalid within-object trial), or at the homologous end of the other rectangle (invalid between-object trial). As shown in Figure 1, the experimental design was fully balanced, such that invalid cueing could require attention to be redirected either between (or within) rectangles equally frequently between or within visual hemifields. The cue position relative to the fixation point was equidistant at $6^{\circ}$ horizontal/vertical, in all presentation conditions. The target remained on-screen for $3000 \mathrm{~ms}$ or until response (pressing of the space bar), whichever was sooner. This terminated the trial; the next one started after a $500 \mathrm{~ms}$ inter-trial interval during which the screen was blank (see Fig. 1).

There were 352 trials in total, comprising 256 valid trials, 64 invalid trials, and 32 catch trials with no target present. For each array type (i.e. horizontal or vertical rectangles) there were 176 trials, in 160 of which the target was present. This resulted in 40 (32 valid and 8 invalid) target-present trials for each end of a given (horizontal or vertical) rectangle. Thus, for each rectangle there were 8 invalid targets at the opposite end of the same rectangle and 8 invalid targets at the homologous end of the opposite rectangle. Not taking into account the catch trials, the cue validity was $75 \%$ for each end of a cued rectangle.

Figure 1 about here 


\title{
Experimental design
}

A crossover design was used whereby each subject took part in two testing sessions. Half of the subjects in each group performed the attentional task after sham adaptation followed by prism adaptation. The other half received the same conditions in reverse order. The order of conditions was counterbalanced within each group and each condition was performed with a one-week interval between them to minimize carryover effects. In addition, each experimental session was split into two blocks of trials each preceded by an adaptation period in order to ensure a lasting prismatic aftereffect for the duration of the session (Fig. 2).

Figure 2 about here

\begin{abstract}
Results
The analyses were carried out on group means based on each individual's median reaction times. Before calculating the medians, however, the raw RT data were trimmed to remove outliers more than 3 SDs above each subject's overall mean. Overall, between $0.6 \%$ and $2.5 \%$ of all trials were omitted from the analyses. All difference calculations (invalid-valid) were carried out on individual median RTs.
\end{abstract}

\section{Main analyses}

Figure 3 summarizes the RT performance for each group (HC, RBD, NEG), broken down by adaptation condition (sham, prism), cue validity (valid, invalid within-object, invalid between-object) and target side (left, right). An initial ANOVA found a highly significant four-way interaction between these four factors $[\mathrm{F}(4,58)=9.34, \mathrm{p}<$ 
0.0001]. This was followed up by a series of repeated measures ANOVAs within each subject group, with adaptation condition, cue validity and target side as factors, and Huyn-Feldt adjustments to the degrees of freedom for violations of sphericity.

Figure 3 about here

For the HC group (Fig. 3, left column), significant main effects of validity condition $[\mathrm{F}(1.09,11.95)=55.04, \mathrm{p}<0.0005]$ and side $[\mathrm{F}(1,11)=6.25, \mathrm{p}<0.05]$ were found. Notably, adaptation condition had no influence on performance in this group. The 'side' effect reflected slightly longer RTs to left-side targets overall (mean RT $510 \mathrm{~ms}$ left, $503 \mathrm{~ms}$ right). The validity effect reflected the expected cost for invalidly cued relative to validly cued targets, with an additional cost when the invalidly cued target required a between-object attention shift (mean valid RT 415 ms; invalid within-object RT 533 ms; invalid between-object RT $571 \mathrm{~ms}$ ). Bonferroni ttests confirmed significant differences between all pairings of validity conditions (adjusted $\mathrm{p}<0.0005$ in all cases).

The RBD group (Fig. 3, middle column) similarly showed significant main effects of validity $[\mathrm{F}(2,18)=42.08, \mathrm{p}<0.0001]$ and side $[\mathrm{F}(1,9)=7.12, \mathrm{p}<0.05]$, with no significant effects involving adaptation condition. As before, the 'side' effect reflected longer RTs to left-side targets (mean RT $698 \mathrm{~ms}$ left, $652 \mathrm{~ms}$ right), this larger difference no doubt arising as a generalized effect of the right-hemisphere damage in these patients. The validity effect again reflected the cost of invalid cueing and an additional cost for a between-object attention shift (mean RT 552 ms valid, $713 \mathrm{~ms}$ invalid within-object, $761 \mathrm{~ms}$ invalid between-object). Bonferroni t-tests 
confirmed significant differences between all pairings of validity conditions (adjusted $\mathrm{p}<0.05$ in all cases)

As in the two control groups, the analysis for the NEG group (Fig. 3, right column) also found significant main effects of target side $[\mathrm{F}(1,9)=19.29, \mathrm{p}<0.005]$ and validity $[\mathrm{F}(1.21,10.86)=55.97, \mathrm{p}<0.0005] . \quad$ The neglect patients were considerably slower to respond to targets on the left than on the right, slower to respond to invalidly-cued than to validly-cued targets, and slower to make betweenobject than within-object shifts of attention. In contrast to the other two groups, however, all effects involving the factor of adaptation were also highly significant, including the three-way interaction between adaptation condition, validity, and target side $[\mathrm{F}(1.44,12.95)=17.54, \mathrm{p}<0.0005]$.

This result was followed up by separate repeated-measures ANOVAs for each adaptation condition separately. In the sham condition (Fig. 3, upper right plot), the main finding was a pronounced interaction of validity by side $[\mathrm{F}(1.31,11.83)=$ 11.29, $\mathrm{p}<0.005]$, with a greatly elevated RT cost $(657 \mathrm{~ms})$ when attention had to be reoriented between objects to an invalidly-cued target on the left side. The NEG group thus showed the classic disengage deficit for invalidly-cued targets on the left side. This deficit, however, was specific to between-object shifts of attention, and did not affect within-object shifts. Table 2 presents these data in the form of difference scores between within and between-object invalid RTs in the three groups of subjects. The selectivity of the disengage deficit in the neglect group following sham adaptation, and its disappearance following real prism adaptation, are clearly apparent.

Table 2 about here 
In the prism adaptation condition, however, performance in the NEG group conformed to the patterns observed in the two control groups, with significant effects of validity $[\mathrm{F}(2,18)=50.38, \mathrm{p}<0.0005]$ and side $[\mathrm{F}(1,9)=9.72, \mathrm{p}<0.05]$. RTs were longer for left-sided targets (mean RT 814 ms left, $692 \mathrm{~ms}$ right). The validity effect again reflected the cost of invalid cueing and an additional cost for between-object attention shifts (mean RT $582 \mathrm{~ms}$ valid, $803 \mathrm{~ms}$ invalid within-object, $879 \mathrm{~ms}$ invalid between-object). Bonferroni t-tests confirmed significant differences between all pairings of validity conditions (adjusted $\mathrm{p}<0.05$ in all cases). In other words, prism adaptation restored performance in the NEG group to a remarkably normal pattern, abolishing the disengage deficit entirely.

\section{Analysis of valid trials}

The difference between the NEG patients and the control groups is readily evident in terms of the disengage deficit, as is the effect of prism adaptation on this aspect of the NEG group's performance. However, we were also interested in the patients' more fundamental ability to detect validly-cued targets. For this purpose, we performed an additional analysis of reaction times to valid targets with group, adaptation condition and target side as factors. This revealed no significant effects involving adaptation condition, but found a highly significant interaction of group by side $[\mathrm{F}(2,29)=$ 11.20, $\mathrm{p}<0.0005]$. The source of this interaction was investigated in a series of independent t-tests between pairs of groups conducted on difference scores (left minus right RTs to validly-cued targets). Only the difference between the NEG and HC group survived Bonferroni adjustment for multiple comparisons [ $t(9.20)$ with unequal variances $=4.46$, adjusted $\mathrm{p}<0.01]$. Thus the tendency for longer RTs to left-sided targets was more pronounced in the NEG group than in healthy controls, but 
not reliably more pronounced than in RBD controls. In all cases, however, it is clear that right prism adaptation did not induce any differential speeding of responses to validly-cued targets on the left side.

\section{Horizontal vs vertical attention shifts}

In order to evaluate whether the observed attentional asymmetries were hemispatial or directional in nature we reanalysed the raw data by calculating for each subject their median invalidly-cued RTs according to whether attention had to be shifted horizontally (between-hemifield) or vertically (within-hemifield). The valid RTs for the relevant hemifield were subtracted to provide RT costs for the two kinds of shift separately, as shown in Fig. 4.

Figure 4 about here

An ANOVA on these costs with the between-subjects factor 'group' (HC, RBD, NEG) and within-subject factors 'adaptation' (sham, prism), 'attention shift' (within-, between-hemifield) and 'target side' (left, right) revealed a highly significant main effect for 'shift' $[\mathrm{F}(1,29)=24.35, \mathrm{p}<0.0001]$, reflecting the generally higher within-hemifield than between-hemifield costs. More interestingly, there was also a significant 3-way interaction of shift $x$ side $x$ group $[F(2,29)=5.61, p<0.05]$, and a 4way interaction of adaptation $x$ shift $x$ side $x$ group $[F(2,29)=6.74, p<0.005]$.

To explore these interactions, separate ANOVAs were carried out for each group separately. In the NEG group only, these analyses revealed a significant 2-way ( shift $\mathrm{x}$ side) interaction $[\mathrm{F}(1,9)=17.64, \mathrm{p}<0.005]$, and a significant 3-way (adaptation $\mathrm{x}$ shift $\mathrm{x}$ side) interaction $[\mathrm{F}(1,9)=22.78, \mathrm{p}<0.005)]$. No significant 
interactions were found in either of the two control groups. The shift $\mathrm{x}$ side interaction in the NEG group, as Fig. 4 (top right) illustrates, reflects a much more prominent disengage deficit in the NEG group for between-hemifield than for withinhemifield trials after sham adaptation. The 3-way interaction reflects the fact that this selective disengage deficit was abolished by prism adaptation.

We confirmed these interpretations of the NEG group data by performing $2 \mathrm{x}$ 2 ANOVAs on the horizontal and vertical shifts separately. These showed that the main effect of side was significant only for horizontal shifts $[F(1,9)=6.06, p<0.05]$, and not for vertical shifts considered alone. In other words, the disengage deficit was specifically a directional deficit: there was no deficit for vertical attention shifts executed within the left hemifield alone. Similarly, the condition x side interaction was significant only for horizontal shifts $[F(1,9)=14.17, p<0.05]$, reflecting the disappearance of the disengage deficit after prism adaptation.

\section{Figure 5 about here}

\section{Extent of prismatic after-effects}

As shown in Figure 5 (top), the NEG patients showed a substantially larger visuomotor deviation following prism adaptation than either of the two control groups. This difference between groups was significant on a two-way ANOVA $[\mathrm{F}(2,29)=7.82, \mathrm{p}<0.01]$. The ANOVA also revealed that unlike either control group, the neglect patients maintained this strong after-effect between the beginning and the end of the testing session [interaction group $x$ occasion: $F(2,29)=6.76, p<$ 0.01]. We also examined the relationship between this reaching bias after prismatic adaptation and the extent of improvement in the disengage deficit that followed it. As 
illustrated in Figure 5 (bottom), the neglect patients showed a strong correlation between the visuomotor bias and the reduction in between-object disengage deficit between sham and prism sessions $[\mathrm{r}(9)=0.76 ; \mathrm{p}<0.01$, using an averaged reaching bias across initial and final scores]. This correlation did not reach significance in either of the other two groups $(\mathrm{r} \leq 0.33, \mathrm{p}>0.05)$. Finally, there was no significant correlation between the initial (sham) disengage deficit and its improvement following prism adaptation across the neglect patient group, indicating that the prismatic benefit was not influenced by the severity of a patient's deficit.

\section{Discussion}

The task used in the present study elicited attention shift costs somewhat larger than those reported by Egly et al. (1994): our healthy subjects responded to invalidly cued stimuli 137 ms slower than their responses to validly cued stimuli. Nonetheless, just as in that previous study, significantly greater costs were observed (156 ms) for shifts of attention between objects than for shifts within objects (119 ms). These costs in our healthy control group were unaffected by prism adaptation. This normal pattern was replicated in the control group of right-hemisphere patients without neglect: they showed between-object shift costs of $209 \mathrm{~ms}$, as compared with their within-object costs of $161 \mathrm{~ms}$. There was no significant difference in the costs for responding to target stimuli presented in the left versus right visual field. Although numerically these shifting costs in non-neglect patients were higher than those observed in our healthy controls, their reaction times were longer. Proportionately, their shift costs were almost identical to those of the healthy subjects. Following prism adaptation, they too showed no significant change in attention-shifting costs on either task. 
Our neglect patients showed a completely different pattern of results from the other two groups. At baseline, that is following sham adaptation only, they exhibited an extremely large RT cost for leftward attention shifts between objects. Yet their costs for rightward between-object shifts, and for within-object shifts, whether leftward or rightward, remained proportionately comparable to those seen in the lesion controls (given that their mean RTs were even longer). Furthermore, there was no difference between the within- and between-object conditions for rightward shifts of attention.

Following prism adaptation, the pattern of RT costs in the neglect patients came close to the normal pattern seen in the two control groups. That is, there was now a completely symmetrical pattern in which between-object shifts had higher costs (301 ms) than within-object shifts (225 ms). Allowing for the longer RTs made by our neglect patients, this pattern thus became relatively normal in percentage terms, i.e. the costs observed were now comparable to the baseline (sham-adaptation) results from our two control groups.

Our experimental design, following that of Egly et al. (1994), included vertical as well as horizontal shifts of attention, balanced across both types of invalid-cue condition. It was therefore possible to compare the effects of shifting attention within one visual hemifield with those of shifting attention between hemifields (averaging across both between- and within-object cueing). Our data show that such vertical attention shifts were overall somewhat more costly (by, on average, $47 \mathrm{~ms}$ ) in both of our control groups, in both hemifields. This was true also in our neglect patients when the target stimulus appeared in the right hemifield. Only when the target stimulus appeared in the left field was a different pattern obtained. Prior to prism adaptation, the neglect patients now showed the opposite result: a significantly higher 
cost for right-to-left attention shifts than for upward or downward shifts. In other words, their disengage deficit was clearly restricted to lateral attention shifts from the right to left hemifield. Following prism adaptation, this pattern reversed dramatically to the 'normal' pattern of a higher cost for vertical than for horizontal shifts to lefthemifield targets.

In our sample of neglect patients, the disengage deficit was observed only for between-object attention shifts. This seems to indicate that the disengage deficit is strongly object-based, and not purely spatial: if the disengage deficit were spacebased only, then we it should be equally apparent in the within- and between-object conditions, because the spatial factors are the same in both cases. In fact, the perceptual chunking of the scene into objects had a huge influence on the disengage deficit. This is consistent with a recent report by Rastelli et al. (2008), who observed that the disengage deficit in neglect was abolished by the use of a peripheral offset cue, as opposed to an onset cue. These authors concluded that the disengage deficit depends critically upon the presence of an object from which attention must be disengaged, and does not apply to locations in space per se. To this conclusion, we may now add that the presence of an object on which attention is engaged may impede the reorienting of attention to contralesional locations away from that object, but it does not does impede reorienting within the same object.

At face value, our findings seems to conflict with the previous data of Egly and colleagues (1994), who concluded in favour of an object-based component to the disengage deficit in left-hemisphere but not right-hemisphere patients with parietal involvement. However, it may not be possible to make such direct comparisons between Egly et al's data and our own, because they used unselected groups of left and right-hemisphere patients with some degree of parietal involvement, not all of 
whom had neglect. In contrast, not all of our patients had parietal involvement, even among the neglect group. In other words, the baseline data for our patients complement, and do not necessarily contradict, those of Egly et al. More specifically, our data speak to the nature of spatial neglect, but not directly to the nature of parietal lobe function per se. Indeed recent evidence suggests that the superior temporal region may have at least as much to do with the attentional functions we are addressing in this study (Karnath et al. 2001, 2004; Ellison et al. 2004; Schindler et al. 2008) as does the parietal lobe (Mort et al. 2003; Rorden et al. 2006). Finally of course, all of our patients, unlike those of Egly et al, had right-hemisphere infarcts, and therefore our data cannot contribute to the question of hemispheric asymmetries in attentional control.

The experimental task that we adopted was designed by Egly et al. (1994) to contrast object-based and space-based components of reorienting; it was not designed explicitly to test for 'within-object' forms of neglect. Nonetheless, the task should be sensitive to such symptoms, in that a hypothetical patient who has a within-object but not a between-object attentional bias should show greater RT costs when an invalidly cued target appears within the cued object, rather than the other object. In reality, we found that within-object biases made little if any contribution to neglect performance. First, at a group level, within-object reorienting of attention to invalidly cued contralesional targets was remarkably efficient. Second, none of our neglect patients had a greater RT cost for within-object than for between-object reorienting to leftsided targets, as would be expected for any patient with a within-object form of neglect. This apparent absence of within-object biases in the attentional task occurred despite clear neglect on clinical tasks, such as figure copying and drawing (see Table 1), that are sometimes held to be diagnostic of within-object forms of neglect. This 
adds weight to earlier warnings that caution is required in inferring within-object neglect from uncontrolled clinical tests such as these, since more parsimonious accounts may explain within-object patterns of errors (e.g. Driver and Pouget 2000).

Indeed, whilst there is no doubt that object-based effects can powerfully shape the symptoms of neglect (e.g. Driver and Halligan, 1991; Behrmann and Tipper, 1994; Walker, 1996), evidence for a truly dissociable within-object form of neglect is limited to a pair of single-case studies (e.g. Humphreys and Riddoch 1994). No previous study has assessed the incidence of within-object effects in unselected patients with neglect, so we really do not know how common such effects may be, or to what extent they contribute to the clinical presentation of neglect. The lack of evidence for 'within-object' neglect in the present study suggests that such effects may be rare at best. On the other hand, the absence of within-object effects may reflect something specific about our attentional task, thus not ruling out the possibility that some of our patients might have shown within-object neglect in other tests. However, at least with regard to the disengage deficit, our data establish a vastly greater impairment when making attention shifts between objects than within them.

Analysis of the extent of the visuomotor after-effect of prism adaptation within the neglect group provided some potentially useful information for understanding the effects of adaptation in these patients. First, the initial amplitude of post-adaptation bias was $35 \%$ greater in the neglect group than in the other two groups; and this larger effect was maintained throughout the test session, whereas the bias fell by $14 \%$ in the controls. These results confirm previous reports that neglect patients show abnormally large prismatic after-effects which also decay more slowly than normal (Rossetti et al. 1998; Serino et al. 2006). Given that no similar effects were seen in our RBD controls, it appears that these large and persistent after-effects 
are truly specific to neglect. Secondly, our analyses also uncovered a strong relationship between the extent of post-prismatic visuomotor bias and the improvement in disengage deficit observed among our neglect patients. This finding contrasts with a previous report (Serino et al. 2006) that there is no such relationship between prismatic after-effect and the degree of recovery from neglect. It may be that the reason for this apparent disagreement lies in the selection of neglect tests used: in order to detect the relationship it may be necessary to use a very pure and specific measure of attentional bias. It should be cautioned that the visuomotor biases we were able to analyse do not provide a pure measure of the visuomotor after-effects, given that we did not have a pre-adaptation baseline measure of pointing error. This may mean that we have tended to underestimate the adaptation effects in our analyses. However we do not believe that this would have affected our conclusions. In particular the lack of such a baseline does not compromise our demonstration of the stability of the after-effect in the neglect patients.

As mentioned in the Introduction, there have been two recent reports on the effects of prism adaptation on the performance of neglect patients in attentional tasks using the Posner paradigm. In particular, Nijboer et al. (2008) have argued from data based on two patients that voluntary attention shifts benefit from adaptation while automatic shifts do not. This was based on the finding of an effect with central symbolic predictive cueing, and long SOAs, but no effect with peripheral nonpredictive cueing and short SOAs. The present study, however, establishes that it cannot be the difference between central and peripheral cues per se that is critical, because we have reported here a massive effect of prism adaptation in a task with peripheral cues. The difference may instead be that, in the present task, the peripheral cues were informative, and therefore would have encouraged voluntary as well as 
engaging automatic orienting processes. It is notable that a neuroimaging study by Kincade et al. (2005) indicates that the "ventral attention network" including the temporoparietal junction (TPJ), may be especially involved in stimulus-driven shifts of attention to task-relevant stimuli at unattended locations (i.e. attentional reorienting). Moreover, they found that the ventral network (especially TPJ) is much more strongly activated when attention has initially been directed endogenously. These data suggest that the TPJ, an area frequently damaged in neglect patients, may be a critical centre for stimulus-driven reorienting following endogenous orienting. It may be that such reorienting is the aspect of attention that benefits most directly from prism adaptation in neglect patients.

The effects of prism adaptation on our neglect patients in this study were every bit as dramatic as previous reports have led one to expect (Rossetti et al. 1998; Rossetti and Rode 2002). They were also apparently rather selective, in that they affected only leftward shifts of attention between objects. This conclusion, however, has to be tempered by the fact that it was only such leftward between-object shifts that were significantly impaired in our neglect patients in the first place. In other words, it could be argued that in our sample there was no deficit in within-object attentionshifting to be corrected. There is thus an ambiguity in the present data. Is it that prism adaptation benefits are truly restricted to between-object shifts of attention in neglect patients, such that a within-object task that showed an impairment would also remain unaffected? Or would any task that showed a leftward reorienting impairment in neglect show a comparable improvement following prism adaptation? On the basis of the present data, we wish to argue that within this type of Posner cueing paradigm, there is an attentional impairment (disengage deficit) commensurate with the severity of the patients' clinical neglect symptoms only for attention shifts between objects. 


\section{Acknowledgements}

The authors are grateful to the Medical Research Council (grants G0000003 and G0000680) and the Leverhulme Trust (grant F/00128/O) for their support. 


\section{References}

Behrmann M, Tipper SP (1994) Object-based attentional mechanisms: Evidence from patients with unilateral neglect. In: Umiltà C, Moscovitch M (eds) Attention and Performance XV. Conscious and Nonconscious Information Processing. MIT Press, Cambridge, MA., pp 351-375

Berberovic NN, Pisella L, Morris AP, Mattingley JB (2004) Prismatic adaptation reduces biased temporal order judgements in spatial neglect. (2004) Prismatic adaptation reduces biased temporal order judgements in spatial neglect. Neuroreport 15: 1199-1204

Driver J, Halligan PW (1991) Can visual neglect operate in object-centred coordinates? An affirmative single case study. Cogn Neuropsychol 8: 475-496

Edgworth J, Robertson IH, MacMillan T (1998) The Balloons Test: A Screening Test for Visual Inattention. Thames Valley Test Company, Bury St Edmunds, UK

Egly R, Driver J, Rafal RD (1994) Shifting visual attention between objects and locations: evidence from normal and parietal lesion subjects. J Exp Psychol Gen 123: 161-177

Ellison A, Schindler I, Pattison LL, Milner AD (2004) An exploration of the role of the superior temporal gyrus in visual search and spatial perception using TMS. Brain 127: 2307-2315

Frassinetti F, Angeli V, Meneghello F, Avanzi S, Ladavas E (2002) Long-lasting amelioration of visuospatial neglect by prism adaptation. Brain 125: 608-623.

Friedrich FJ, Egly R, Rafal RD, Beck D (1998) Spatial attention deficits in humans: a comparison of superior parietal and temporal-parietal junction lesions.

Neuropsychology 12: 193-207 
Gainotti G, Messerli P, Tissot, R (1972) Qualitative analysis of unilateral spatial neglect in relation to laterality of cerebral lesions. J Neurol Neurosurg Psychiat 35: 545-550.

Humphreys GW, Riddoch MJ (1994) Attention to within-object and between-object spatial representations - multiple sites for visual selection. Cogn Neuropsychol 11: 207-241

Karnath H-O, Ferber S, Himmelbach M (2001) Spatial awareness is a function of the temporal not the posterior parietal lobe. Nature 411: 950-953

Karnath H-O, Fruhmann Berger M, Kuker W, Rorden C (2004) The anatomy of spatial neglect based on voxelwise statistical analysis: a study of 140 patients. Cereb Cortex 14: 1164-1172

Kincade JM, Abrams RA, Astafiev SV, Shulman GL, Corbetta M (2005) An eventrelated functional magnetic resonance imaging study of voluntary and stimulus-driven orienting of attention. J Neurosci 25: 4593-4604

Kleinman JT, Newhart M, Davis C, Heidler-Gary J, Gottesman RF, Hillis AE. (2007) Right hemispatial neglect: frequency and characterization following acute left hemisphere stroke. Brain \& Cognition 64: 50-59.

Ladavas E, Carletti M, Gori G (1994) Automatic and voluntary orienting of attention in patients with visual neglect: horizontal and vertical dimensions. Neuropsychologia 32: 1195-1208

Losier BJ, Klein RM (2001) A review of the evidence for a disengage deficit following parietal lobe damage. Neurosci Biobehav Rev 25: 1-13

McIntosh RD, Rossetti Y, Milner AD (2002) Prism adaptation improves chronic visual and haptic neglect: a single-case study. Cortex 38: 309-320

Milner AD, McIntosh RD (2005) The neurological basis of visual neglect. Current 
Opin Neurol 18: 748-753

Morris AP, Kritikos A, Berberovic N, Pisella L, Chambers CD, Mattingley JB (2004) Prism adaptation and spatial attention: a study of visual search in normals and patients with unilateral neglect. Cortex 40: 703-721

Morrow LA, Ratcliff G (1988) The disengagement of covert attention and the neglect syndrome. Psychobiol 3: 261-269

Mort DJ, Molhotra P, Mannan SK, Rorden C, Pambakian A, Kennard C, Husain M (2003) The anatomy of visual neglect. Brain 126: 1986-1997

Nijboer, TCW, McIntosh RD, Nys GMS, Dijkerman HC, Milner AD (2008) Prism adaptation improves voluntary but not automatic orienting in neglect. Neuroreport 19: 293-298

Posner MI (1980) Orienting of attention. Quart J Exp Psychol 32: 3-25

Posner MI, Cohen Y, Rafal RD (1982) Neural systems control of spatial orienting. Phil Trans R Soc Lond, B 298: 187-198

Posner MI, Walker JA, Friedrich FJ, Rafal RD (1984) Effects of parietal lobe injury on covert orienting of attention. J Neurosci 4: 1863-1874

Posner MI, Walker JA, Friedrich FA, Rafal RD (1987) How do the parietal lobes direct covert attention? Neuropsychologia 25: 135-145

Rastelli F, Funes M-J, Lupiáñez J, Duret C, Bartolomeo P (2008) Left visual neglect: is the disengage deficit space- or object-based? Exp Brain Res 187: 439-446.

Redding GM, Wallace B (2006) Prism adaptation and unilateral neglect: review and analysis. Neuropsychologia 44: 1-20

Rode G, Rossetti Y, Boisson D (2001) Prism adaptation improves representational neglect. Neuropsychologia 39: 1250-1254

Rode G, Rossetti Y, Li L, Boisson D (1999) Improvement of mental imagery after 
prism exposure in neglect: a case study. Behav Neurol 11: 251-258

Rorden C, Fruhmann Berger M, Karnath H-O (2006) Disturbed line bisection is associated with posterior brain lesions. Brain Res 1080: 17-25

Rossetti Y, Rode G (2002) Reducing spatial neglect by visual and other sensory manipulations: noncognitive (psychological) routes to the rehabilitation of a cognitive disorder. In: Karnath H-O, Milner AD, Vallar G (eds) Cognitive and Neural Bases of Spatial Neglect. Oxford University Press, Oxford, pp 375-396

Rossetti Y, Rode G, Pisella L, Farnè A, Li L, Boisson D, Perenin MT (1998) Prism adaptation to a rightward optical deviation rehabilitates left hemispatial neglect. Nature 395: 166-169

Rossetti Y, Rode G, Pisella L, Farne A, Ling L, Boisson D (1999) Sensorimotor plasticity and cognition: prism adaptation can affect various levels of space representation. In: Grealy M, Thomson JA (eds) Studies in Perception and Action. Lawrence Erlbaum Associates, Hove, UK, pp 265-269

Schindler I, Ellison A, Milner AD (2008) Contralateral visual search deficits following TMS. J Neuropsychol: published online 2 Feb 2008

Striemer C, Danckert J (2007) Prism adaptation reduces the disengage deficit in right brain damage patients. Neuroreport 18: 99-103

Tilikete C, Rode G, Rossetti Y, Pichon J, Li L, Boisson D (2001) Prism adaptation to a rightward optical deviation improves postural imbalance in left-hemiparetic patients. Curr Biol 11: 1-5

Walker R (1996) Spatial and object-based neglect. Neurocase 1: 371-383

Wilson B, Cockburn J, Halligan PW (1987) The Behavioural Inattention Test. Thames Valley Test Company, Titchfield, Hampshire, UK 
Table 1: Clinical and demographic data of the neglect (NEG) and control (RBD) patients.

\begin{tabular}{|c|c|c|c|c|c|c|c|c|c|c|}
\hline \multirow[t]{2}{*}{ Patient } & \multirow[t]{2}{*}{ Age/sex } & \multirow[t]{2}{*}{$\begin{array}{l}\text { Months } \\
\text { post onset }\end{array}$} & \multirow[t]{2}{*}{ Aetiology } & \multirow[t]{2}{*}{$\begin{array}{l}\text { Lesion site } \\
\text { (CT scan) }\end{array}$} & \multicolumn{2}{|c|}{$\begin{array}{l}\text { Star cancellation } \\
\text { Omissions }(\%)\end{array}$} & \multirow[t]{2}{*}{$\begin{array}{c}\text { Balloons B } \\
\text { Laterality index (\%) }\end{array}$} & \multirow[t]{2}{*}{$\begin{array}{l}\text { BIT line bisection } \\
\text { Mean deviation }(\mathrm{mm})\end{array}$} & \multirow[t]{2}{*}{$\begin{array}{l}\text { Scene copying } \dagger \\
\text { Symmetry/Items }\end{array}$} & \multirow[t]{2}{*}{ Drawing } \\
\hline & & & & & $\mathrm{L}$ & $\mathrm{R}$ & & & & \\
\hline NEG1 & $75 / \mathrm{m}$ & 33 & Ischaemia & $\mathrm{TP}, \mathrm{WM}, \mathrm{SC}$ & 7.4 & 3.7 & 37.5 & 15.5 & $2 / 4$ & 3 \\
\hline NEG2 & $75 / f$ & 15 & Haemorrhage & $\mathrm{P}$ & 3.7 & 3.7 & 37.5 & 14.6 & $4 / 5$ & 3 \\
\hline NEG3 & $75 / \mathrm{m}$ & 36 & Ischaemia & FPO & 29.6 & 25.9 & 12.5 & 15.3 & $1 / 1$ & 1 \\
\hline NEG4 & $59 / f$ & 12 & Ischaemia & n. a. & 11.1 & 3.7 & 33.3 & 16.2 & $5 / 5$ & 3 \\
\hline NEG5 & $58 / \mathrm{m}$ & 2 & Ischaemia & $\mathrm{TP}$ & 14.8 & 3.7 & 37.5 & 12.4 & $5 / 5$ & 3 \\
\hline NEG6 & $83 / \mathrm{m}$ & 27 & Ischaemia & $\mathrm{P}$ & 100 & 22.2 & 0 & 7.3 & $2 / 5$ & 0 \\
\hline NEG7 & $75 / f$ & 4 & Ischaemia & $\mathrm{FT}, \mathrm{BG}$ & 3.7 & 0 & 33.3 & 16.5 & $4 / 5$ & 3 \\
\hline NEG8 & $74 / \mathrm{m}$ & 3 & Ischaemia & $\mathrm{FP}$ & 55.6 & 33.3 & 18.2 & 19.1 & $0 / 5$ & $\mathbf{0}$ \\
\hline NEG9 & $50 / \mathrm{m}$ & 2 & Ischaemia & $\mathrm{FP}, \mathrm{BG}$ & 14.8 & 3.7 & 20.0 & 3.9 & $4 / 5$ & 2 \\
\hline NEG10 & $59 / \mathrm{m}$ & 13 & Ischaemia & FT & 100 & 3.7 & 33.3 & -2.1 & $5 / 5$ & 2 \\
\hline RBD1 & $68 / \mathrm{f}$ & 3 & Ischaemia & WM & 0 & 0 & 50.0 & 1.7 & $5 / 5$ & 3 \\
\hline RBD2 & $62 / f$ & 2 & Ischaemia & $\mathrm{SC}, \mathrm{WM}$ & 0 & 0 & 53.9 & -4.7 & $5 / 5$ & 3 \\
\hline RBD3 & $78 / \mathrm{m}$ & 25 & Ischaemia & $\mathrm{BG}$ & 0 & 0 & 50.0 & 1.2 & $5 / 5$ & 3 \\
\hline RBD4 & $57 / \mathrm{m}$ & 2 & Ischaemia & FT & 0 & 3.7 & 47.1 & -9.4 & $5 / 5$ & 3 \\
\hline RBD5 & $41 / \mathrm{m}$ & 3 & Haemorrhage & $\mathrm{F}$ & 0 & 0 & 50.0 & -1.3 & $5 / 5$ & 3 \\
\hline RBD6 & $69 / \mathrm{m}$ & 7 & Ischaemia & $\mathrm{T}, \mathrm{P}$ & 3.7 & 3.7 & 52.9 & 8.9 & $5 / 5$ & 3 \\
\hline RBD7 & $79 / \mathrm{m}$ & 18 & Ischaemia & $\mathrm{FP}$ & 0 & 0 & 53.3 & 4.0 & $5 / 5$ & 3 \\
\hline RBD8 & $69 / \mathrm{m}$ & 2 & Ischaemia & $\mathrm{T}$ & 0 & 0 & 50.0 & 1.7 & $5 / 5$ & 3 \\
\hline RBD9 & $76 / f$ & 4 & Ischaemia & FT & 3.7 & 3.7 & 50.0 & 0.8 & $5 / 5$ & 3 \\
\hline RBD10 & $69 / \mathrm{m}$ & 6 & Ischaemia & $\mathrm{SC}$ & 0 & 0 & 50.0 & 0.7 & $5 / 5$ & 3 \\
\hline
\end{tabular}

$\uparrow$ Symmetry: total number of attempted items copied symmetrically; Items: total number of items copied (from 5)

+ Drawing from memory of 3 objects (person, clock, daisy): number of items (0-3) drawn symmetrically.

F:frontal, T: temporal, P: parietal, O: occipital, BG: basal ganglia, WM: white matter, SC: subcortical

n.a.: not available.

Test scores that meet the criteria for neglect are shown in bold print. 
Table 2: Mean reaction time cost in ms (SD) for healthy controls (HC), lesion control patients (RBD) and neglect patients (NEG), as a function of adaptation condition, target position (Left, Right) and type of attention shift (within/between object).

\begin{tabular}{|c|c|c|c|c|}
\hline \multicolumn{5}{|c|}{ Sham adaptation } \\
\hline \multirow[t]{2}{*}{ Group } & \multicolumn{2}{|c|}{ Within object } & \multicolumn{2}{|c|}{ Between object } \\
\hline & Left & Right & Left & Right \\
\hline $\mathrm{HC}$ & $124.50(53.98)$ & $115.75(67.94)$ & $169.63(70.31)$ & $149.08(76.04)$ \\
\hline RBD & $110.80(78.08)$ & $187.70(94.54)$ & $240.35(134.38)$ & $207.20(112.89)$ \\
\hline \multirow[t]{2}{*}{ NEG } & $240.00(158.67)$ & $251.80(195.34)$ & $656.80(362.69)$ & $246.10(160.23)$ \\
\hline & \multicolumn{3}{|c|}{ Prism adaptation } & $\bar{s}$ \\
\hline $\mathrm{HC}$ & $122.92(54.60)$ & $111.00(69.75)$ & $154.67(80.34)$ & $150.50(76.40)$ \\
\hline RBD & $164.50(149.26)$ & $179.60(99.20)$ & $184.50(111.58)$ & $202.70(120.91)$ \\
\hline NEG & $223.70(145.82)$ & $226.60(89.59)$ & $299.60(165.60)$ & $302.40(153.21)$ \\
\hline
\end{tabular}


Fig. 1 Timeline for the experimental task. Trials were either validly cued, or invalidly cued either to the same or the other rectangle. Subjects responded to the target as rapidly as possible and reaction times were measured. The two example trials shown illustrate the fact that the two rectangles were presented either vertically or horizontally, in a balanced design. This allowed within vs between-object attention shifts to be distinguished from vertical vs horizontal shifts. See text for more details. $275 \times 190 \mathrm{~mm}(96 \times 96 \mathrm{DPI})$ 

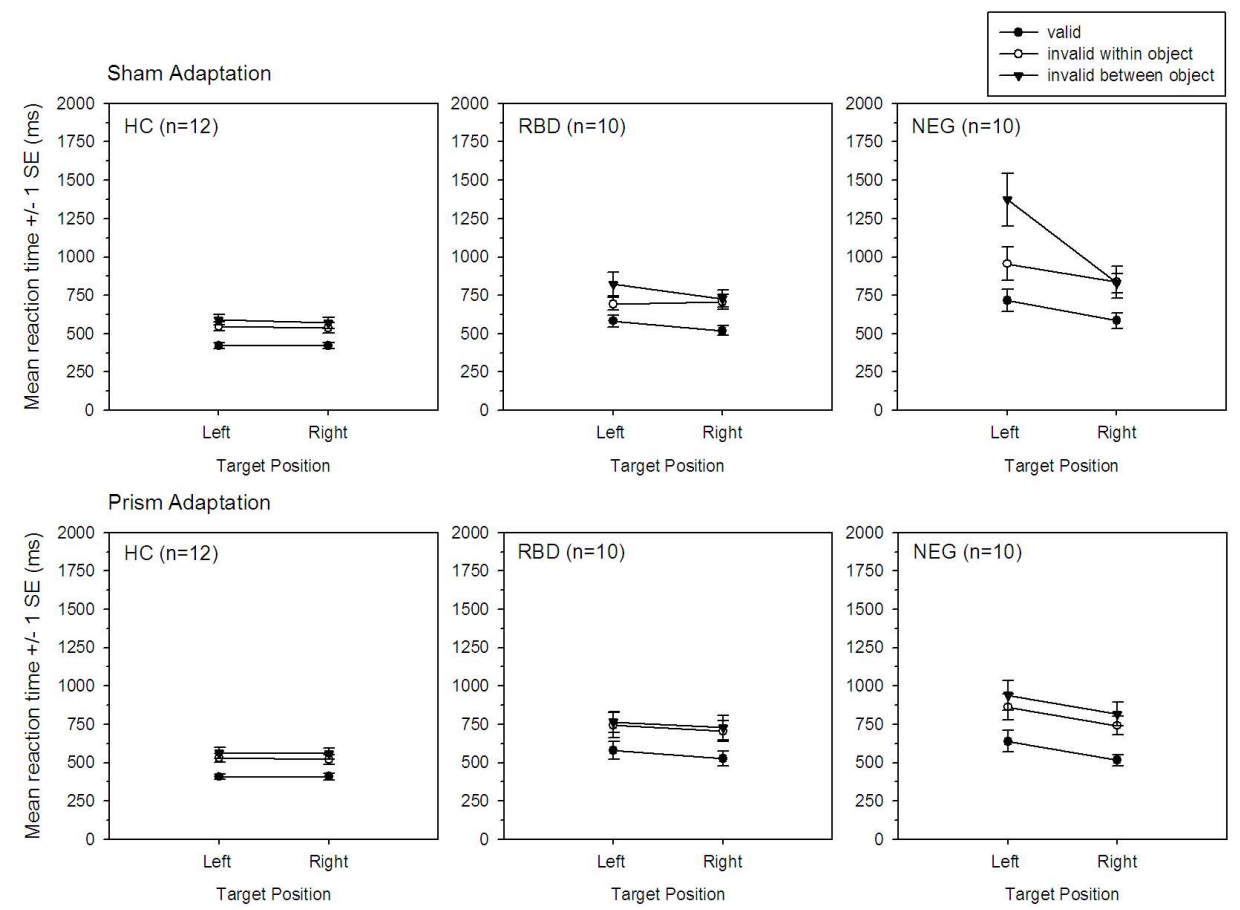

Fig. 3 The reaction time data for three age-matched groups of subjects: neglect patients, right brain damaged controls, and healthy controls. Data are presented separately for the conditions of sham adaptation (top) and real prism adaptation (bottom). In each of the 6 plots, RTs are presented separately for left and right target location and for different cueing conditions (valid, between-object invalid, and within-object invalid). $296 \times 209 \mathrm{~mm}(150 \times 150 \mathrm{DPI})$ 
Fig. 4 RT costs plotted according to whether they required a horizontal shift between left and right hemifields or only a vertical shift within one hemifield, for the three groups of subjects. Again the data are presented separately for sham adaptation (top) and real prism adaptation (bottom). In each plot, RT costs are presented separately for left and right target locations.
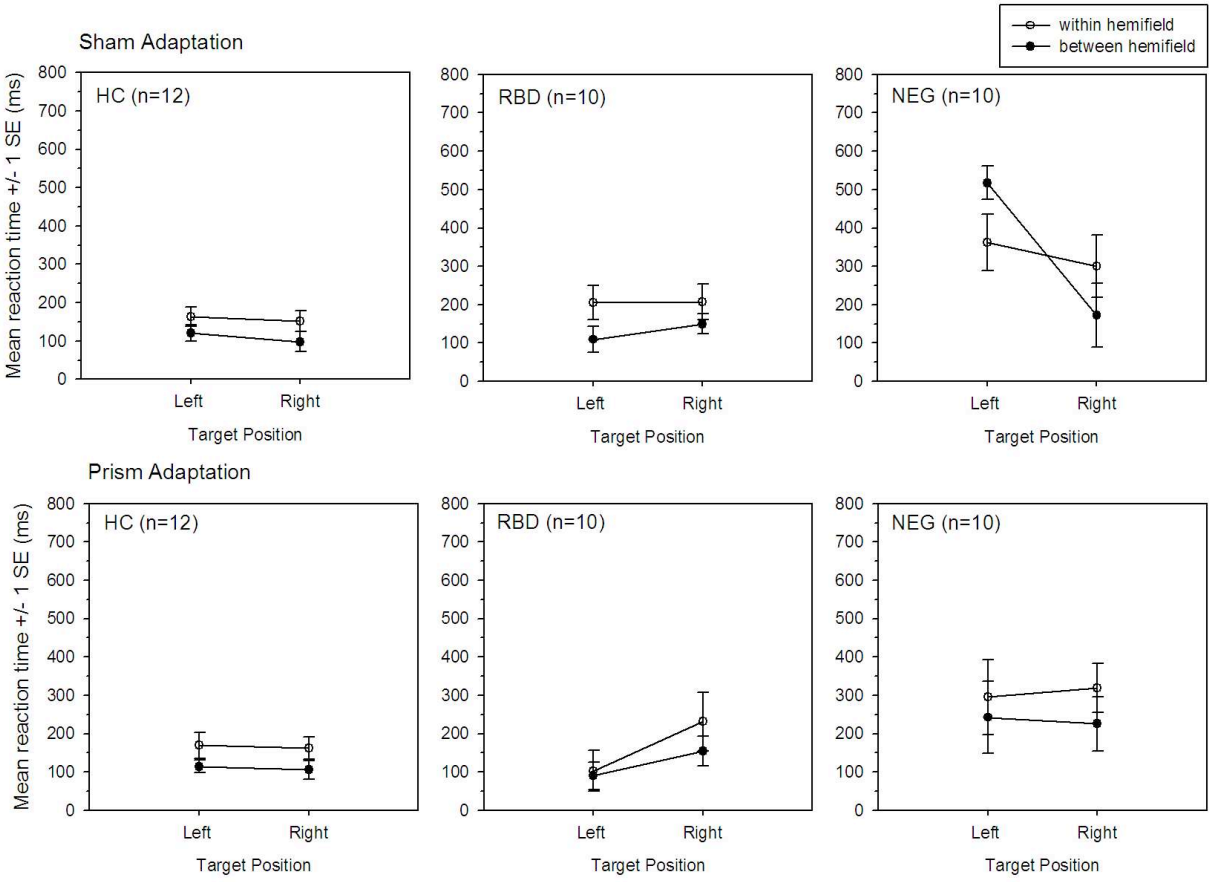
A

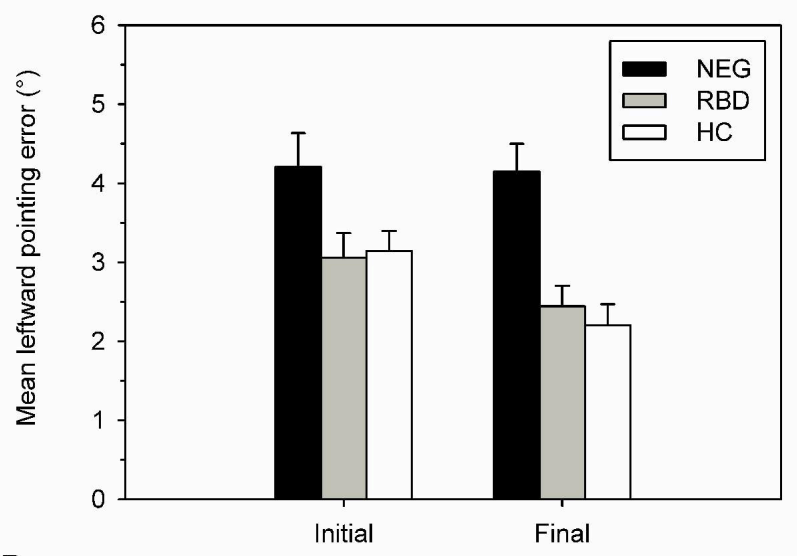

B

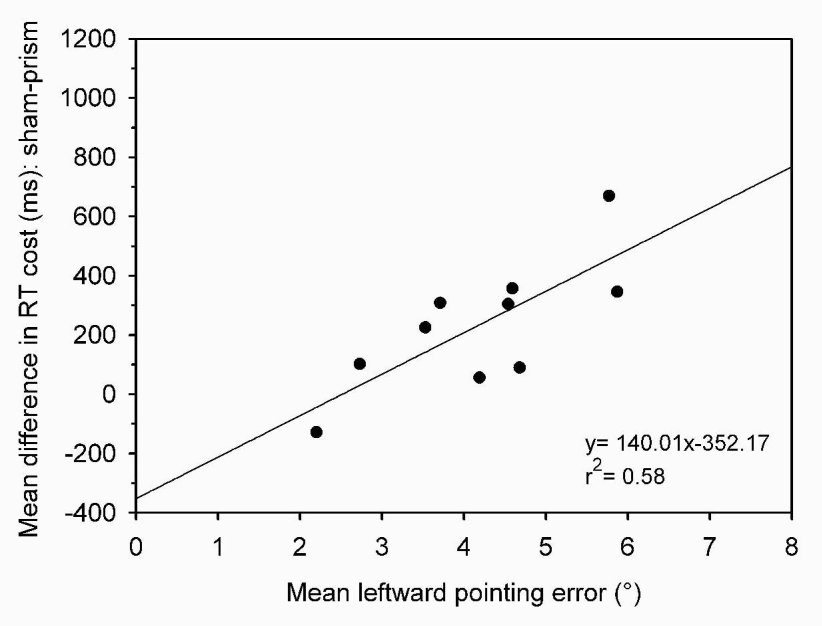

Fig. 5 The extent of the prismatic after-effect and its relationship with the resulting improvements in leftward attention switching. A: Mean pointing error (deg) induced by prism adaptation prior to and following attentional testing in the three groups of subjects. Pointing error was highest and also most stable within the neglect group. B: Scattergram showing the strong relationship between the mean post-prism pointing error in neglect patients and the difference in leftward shift cost between prism and sham test conditions. $839 \times 1187 \mathrm{~mm}(150 \times 150 \mathrm{DPI})$ 